RECYT

Año 22 / № 33 / 2020 / 58-62

\title{
Optimización del diseño e instrumentación del concentrador doble efecto de la Facultad de Ingeniería, Universidad Nacional de San Juan. Argentina
}

\section{Optimization of the design and instrumentation of the double-effect concentrator at School of Engineering. National University of San Juan. Argentina}

\author{
Graciela Ingrid Suarez ${ }^{1,{ }^{*}}$, Veronica Beatriz Navas Nale ${ }^{1}$, Carla Valeria Lorenzo Doncel ${ }^{1}$ \\ 1- Facultad de Ingeniería, Universidad Nacional de San Juan, Argentina. \\ *E-mail: ginsuarez@gmail.com \\ Recibido el 27 de julio de 2018, Aprobado el 25 de noviembre de 2019.
}

\section{Resumen}

En el Laboratorio Tecnológico de la Facultad de Ingeniería, UNS se cuenta con un evaporador doble efecto en escala piloto. El presente artículo analiza el mismo, consolidando conocimientos de operación del concentrador de doble efecto y de instrumentación industrial, estableciendo las variables a manipular y controlar, y realizando mejoras en el diseño, con el objeto de que pueda ser utilizado tanto en investigación como para prácticas de los alumnos de las carreras de Ingeniería en Alimentos, Ingeniería Industrial y de Ingeniería Química. Se trabajó con el equipo, se lo desarmó, se realizaron ensayos y pruebas determinando que la variable a controlar más representativa para el evaporador es la temperatura, se determinaron los puntos en los cuales se colocaron los sensores y como mejoras se determinó la colocación de una válvula y el agregado de dos purgas para poder limpiar el equipo.

Palabras clave: Evaporador; Control; Instrumentación.

\section{Abstract}

The Technological Laboratory at the School Engineering, NUSJ counts with a double-effect evaporator on pilot scale. This article suggests to analyze it by consolidating knowledge about its operational and industrial instrumentation, determining the variables to manipulate it, as well as improving its design and correct use not only on research projects but also in training practices among Food Engineering, Industrial Engineering and Chemical Engineering undergraduates. The equipment was dismantled and several tests were conducted. The results established that the temperature and the placement points of the sensors were the most representative variables for the control of the evaporator. The identified improvements were both the valve placement and the addition of two purges to clean the equipment.

Keywords: Evaporator; Control; Instrumentation.

\section{Introducción}

La Instrumentación y Control, como especialidad de Ingeniería, es la parte de la ingeniería responsable de definir el nivel de automatización de cualquier planta de proceso e instalación industrial, la instrumentación de campo y el sistema de control para un buen funcionamiento del proceso, dentro de la seguridad para los equipos y personas, también de los costos establecidos y manteniendo la calidad.

Por medio de la instrumentación de evaporadores o concentradores, que son utilizados ampliamente en la industria, por ejemplo en la concentración de mosto, se consiguió: analizar las variables del proceso, determinar la más representativa a controlar, se consiguió optimizar recursos, ahorro energético y una calidad de producto constante.

Las últimas cifras dadas a conocer por el Instituto Nacional de Vitivinicultura (INV), Estadísticas de exportaciones, indican que en el año 2016, el volumen de mosto exportado fue de $104.121 \mathrm{tn}$, hubo una disminución del $17,1 \%$ en volumen comparado con el año 2015.

Tabla 1: Exportaciones Argentinas de mosto concentrado (INV).

\begin{tabular}{|c|c|c|c|c|c|c|}
\hline \multirow{2}{*}{ Detalle } & \multicolumn{3}{|c|}{ Toneladas } & \multicolumn{3}{c|}{ Miles de dólares } \\
\cline { 2 - 7 } & $\mathbf{2 0 1 5}$ & $\mathbf{2 0 1 6}$ & $\begin{array}{r}\text { Var. \% } \\
\mathbf{1 6 / 1 5}\end{array}$ & $\mathbf{2 0 1 5}$ & $\mathbf{2 0 1 6}$ & $\begin{array}{c}\text { Var. \% } \\
\mathbf{1 6 / 1 5}\end{array}$ \\
\hline $\begin{array}{c}\text { Mosto } \\
\text { concentrado }\end{array}$ & 120.990 & 99.557 & $-17,7$ & 113.526 & 105.402 & $-7,2$ \\
\hline $\begin{array}{c}\text { Mosto conc. } \\
\text { alcoholizado }\end{array}$ & 4.597 & 4.564 & $-0,7$ & 6.177 & 5.325 & $-13,8$ \\
\hline Total Mostos & 125.588 & 104.121 & $-17,1$ & 119.703 & 110.727 & $-7,5$ \\
\hline
\end{tabular}


Considerando estos antecedentes es que resulta sumamente importante para la industria local como la Argentina la incorporación y mejora de la tecnología en las diferentes etapas de la elaboración de productos concentrados.

\section{Desarrollo}

Analizando el intercambiador de calor según Kern (1999) "Procesos de Transferencia de Calor", Perry (1992) "Manual del Ingeniero Químico y TLV(2012); podemos analizar que por medio de la evaporación podemos eliminar un solvente de un soluto, a través de la generación de una fase gaseosa de un solvente volátil. Para el caso en estudio, a través de un evaporador doble efecto de escala piloto se elimina agua (solvente volátil a la temperatura y presión de trabajo) que es el desecho generado en la operación unitaria del mosto o jugo de uva, dando como producto mosto concentrado (soluto no volátil a temperatura y presión de trabajo).

Se hace referencia a escala piloto considerando que el equipo es de un tamaño reducido es capaz de operar con un pequeño caudal de materia prima a igual condición de presión y temperatura que una planta tamaño industrial.

Este evaporador al ser de doble efecto (el vapor generado en un efecto se aprovecha en el otro efecto como medio de calefacción) permite un mejor aprovechamiento energético, por la disminución de las pérdidas de calor (a causa de la utilización en el $2^{\circ}$ efecto del vapor generado en el $1^{\circ}$ efecto) y la reducción de los costos operativos.

Si los evaporadores se numeran en el sentido en que disminuye la presión, y las presiones y las temperaturas en cada unidad son, respectivamente, $\mathrm{P}_{1}, \mathrm{P}_{2}, \mathrm{y} \mathrm{T}, \mathrm{T}_{2}$ se cumple que: $\mathrm{P}_{1}>\mathrm{P}_{2}$ y $\mathrm{T}_{1}>\mathrm{T}_{2}$.

Para el estudio del evaporador, se tomaron las siguientes suposiciones:

a) El sistema opera en condiciones de flujo y estado estacionarios. Las velocidades de flujo de masa son tales que en ningún evaporador hay acumulación o disminución de disolvente o soluto. Las concentraciones, las presiones y las temperaturas en cada evaporador se mantienen constantes.

b) El mosto no presenta elevación del punto de ebullición, y los efectos de la carga hidrostática son despreciables.

c) La cantidad de calor necesaria para llevar la alimentación desde su temperatura inicial, TF, hasta la temperatura de ebullición, T1, también se considera despreciable, de tal forma que la cantidad de calor proporcionada por el vapor de calefacción en el primer efecto se encuentra como calor latente en el vapor que sale del mismo.

d) Las entalpías de dilución no se toman en consideración.

e) También se desprecia el calor que lleva el mosto, como consecuencia de estar a una temperatura por encima de la de ebullición, cuando pasa de un efecto a otro.

Entonces, la cantidad de calor transmitida en cada efecto en la unidad de tiempo es:

$\mathrm{Qi}=$ Ui Ai $\Delta \mathrm{T}_{\mathrm{i}}$ siendo $\Delta \mathrm{T}_{1}=\mathrm{T}_{\mathrm{VC}}-\mathrm{T}_{1}$ y $\Delta \mathrm{T}_{2}=\mathrm{T}_{1}-\mathrm{T}_{2}$.

Siendo $\mathrm{T}_{\mathrm{VC}}$ la temperatura del vapor condensado que sale del primer efecto.

Se pueden establecer las ecuaciones anteriores con carácter aproximado ya que los errores cometidos con estas suposiciones son cuantitativamente pequeños.

En los evaporadores de doble efecto las áreas son iguales, por lo que se puede escribir que $\mathrm{U}_{1} \cdot \Delta \mathrm{T}_{1}=\mathrm{U}_{2} \cdot \Delta \mathrm{T}_{2}$ donde se deduce que, en ellos, la diferencia de temperatura es, de forma aproximada, inversamente proporcional al valor del coeficiente global de transmisión de calor en el mismo. Si se considera que no hay elevación en el punto de ebullición, se cumplirá:

$$
\Delta T_{1}=\Delta T_{x} \frac{\frac{1}{U_{1}}}{\frac{1}{U_{1}}+\frac{1}{U_{2}}} \quad y \quad \Delta T_{2}=\Delta T_{x} \frac{\frac{1}{U_{1}}}{\frac{1}{U_{1}}+\frac{1}{U_{2}}}
$$

Se define la capacidad de un evaporador como la cantidad de líquido evaporado en la unidad de tiempo. Como la entalpía de vaporización es prácticamente constante en el rango de presiones utilizado, existe una proporcionalidad entre la cantidad de líquido evaporado y la cantidad de calor transmitido.

En un evaporador de dos efectos con la misma superficie de calefacción en cada uno de ellos y con el coeficiente global medio de transmisión del calor, UM, para los mismos, la cantidad de calor total $\left(\mathrm{Q}_{\mathrm{T}}\right)$ transmitida en la unidad de tiempo es: $\mathrm{Q}_{\mathrm{T}}=\mathrm{Q}_{1}+\mathrm{Q}_{2}=\mathrm{A} \cdot \mathrm{UM}\left(\Delta \mathrm{T}_{1}+\Delta \mathrm{T}_{2}\right)$

En un evaporador simple con la misma superficie de calefacción, A, un coeficiente global de transmisión de calor $\mathrm{U}=\mathrm{UM}, \mathrm{y}$ una diferencia de temperatura igual a la diferencia de temperatura total en el doble efecto, la capacidad es: $\mathrm{Q}=$ A.UM. $\Delta \mathrm{T}_{\mathrm{T}}$

Por lo tanto, en un evaporador de doble efectos, la capacidad es la misma que en uno de efecto simple que tenga el mismo coeficiente global, la misma superficie de calefacción que una de las unidades del doble efecto y que opere con una diferencia de temperatura igual a la diferencia total de temperatura con la que opera el sistema de doble efecto. Por tanto, la capacidad por metro cuadrado de superficie en un evaporador de dos efectos es aproximadamente igual a $1 / 2$ veces la capacidad del evaporador de efecto simple.

En cambio, en el sistema de doble efecto se consigue una gran economía, pues, dado que la variación de la entalpía de vaporización al pasar de un efecto a otro es muy pequeña, cuando se condensa $1 \mathrm{~kg}$ de vapor de calefacción se vaporiza en la disolución hirviente aproximadamente un $\mathrm{kg}$ de agua, y así, aumenta la economía dos veces. 
Tabla 2: comparación de consumos energéticos según la cantidad de efectos utilizados.

\begin{tabular}{|c|c|}
\hline $\begin{array}{c}\text { Consumo de vapor para } \\
\text { evaporar } \mathbf{1} \text { kg de agua }\end{array}$ & En kg de vapor \\
\hline Evaporación a simple efecto & 1,1 \\
\hline Evaporación a doble efecto & 0,57 \\
\hline
\end{tabular}

La capacidad de estos sistemas también se ve reducida por la elevación del punto de ebullición de la disolución, pero no así la economía, ya que ésta depende del balance de energía en cada efecto y no de la velocidad de transmisión del calor.

\section{Propiedades del mosto a concentrar}

Algunas de las propiedades más importantes del mosto a concentrar son las siguientes:

Concentración de la disolución: Aunque el mosto que entra en un evaporador puede ser suficientemente diluido y poseer las propiedades del agua, a medida que aumenta la concentración, la densidad y viscosidad de la disolución aumentan, disminuyendo, por tanto, el coeficiente global de transmisión del calor. Si la ebullición es continuada, se puede alcanzar el estado de saturación, en cuyo caso habrá que retirar los cristales formados. Así mismo, es frecuente que con el aumento de concentración aumente también la temperatura de ebullición de la disolución, dificultando el proceso, incluso impidiéndolo, si no se prevé, ya que podría resultar mayor la temperatura de ebullición que la del propio vapor de calefacción.

Formación de espumas: Puede haber presencia de una capa de espuma durante la ebullición. Esta capa de espuma es indeseable porque el vapor formado puede arrastrarla y, por tanto, perderse una cantidad importante de líquido. Las espumas se forman cuando hay una capa de líquido con distinta tensión superficial que el resto de la masa, ocasionada a veces por la presencia de pequeñísimas partículas sólidas o incluso coloidales, habiéndose dedicado un esfuerzo considerable al estudio de las técnicas antiespumantes.

A veces se utilizan métodos químicos, adicionando pequeñas cantidades de reactivos, para disminuir o reducir la formación de espumas. Si se quiere evitar la adición de sustancias extrañas, se pueden eliminar las espumas modificando el diseño del evaporador, con una superficie caliente que rompa la espuma, (muchas se destruyen a alta temperatura), o haciendo pasar el vapor formado a través de unas placas deflectoras, contra las que choca, evitando así el arrastre.

Formación de incrustaciones: Hay sustancias que se denominan de solubilidad invertida, en las que la solubilidad disminuye con la temperatura, y tienden a depositarse sobre las superficies de calefacción. Otra causa es la descomposición térmica por el calor. En estos casos se produce una disminución del coeficiente global de transmisión del calor en el evaporador, y el proceso, se ve, en parte, impedido, siendo necesario parar el evaporador y limpiar los tubos, (con agua, ácidos, álcalis, rascado mecánico, etc.), cosa que a veces es difícil y costosa.

Eliminación del aire, (u otros incondensables): El vapor de agua arrastra siempre aire y otros gases no condensables, los cuales se acumulan en la cámara de calefacción, con lo que disminuye progresivamente el coeficiente de transmisión de calor.

\section{Metodología}

El mosto ingresa al primer efecto ascendiendo por los tubos ubicados en el interior del primer intercambiador de calor o hervidor de flujo ascendente. Este evapora el agua aumentando su concentración como consecuencia del intercambio de calor que se produce entre el vapor latente provisto por una caldera y el mosto que circula por el interior de los tubos.

El concentrador o evaporador, está formado por una zona de calentamiento, una cámara de condensación y una cámara de evaporación.

En la cámara de condensación el vapor de agua eliminado del mosto se transforma en líquido, cediendo su calor latente de condensación el cual es captado en la cámara de evaporación por el mosto a concentrar.

El mosto concentrado en el primer efecto ingresa al segundo mientras que el vapor abandona la cámara de evaporación a la temperatura de ebullición, obteniéndose una solución concentrada o mosto concentrado en este caso.

El vapor que sale del primer efecto ingresa al segundo evaporando o aumentando la concentración del mosto que sale del primer efecto. Si la concentración del mosto es inferior a la requerida existe la posibilidad de recircularlo hacia el primer efecto nuevamente.

El vapor extraído del segundo efecto pasa a un intercambiador tubular horizontal a través de la carcasa en donde es enfriado o condensado por agua que circula por los tubos. Este condensado se extrae con una bomba de vacío.

\section{Análisis de los componentes del concentrador}

Intercambiador de calor tubular o evaporador tubular: es donde se produce la transferencia del calor del vapor al mosto. Consiste en un haz de tubos a través de los cuales circula el mosto y una carcasa exterior por el cual circula el vapor de agua.

Posee circulación forzada ya que el mosto es un líquido viscoso el cual provoca incrustaciones si no se opera con la velocidad de circulación adecuada, además de que afectaría la calidad del mosto concentrado o producto final.

Separador Flash: el mosto al ingresar al separador flash disminuye su presión produciendo la separación entre el mosto concentrado y el vapor que lo constituía. 


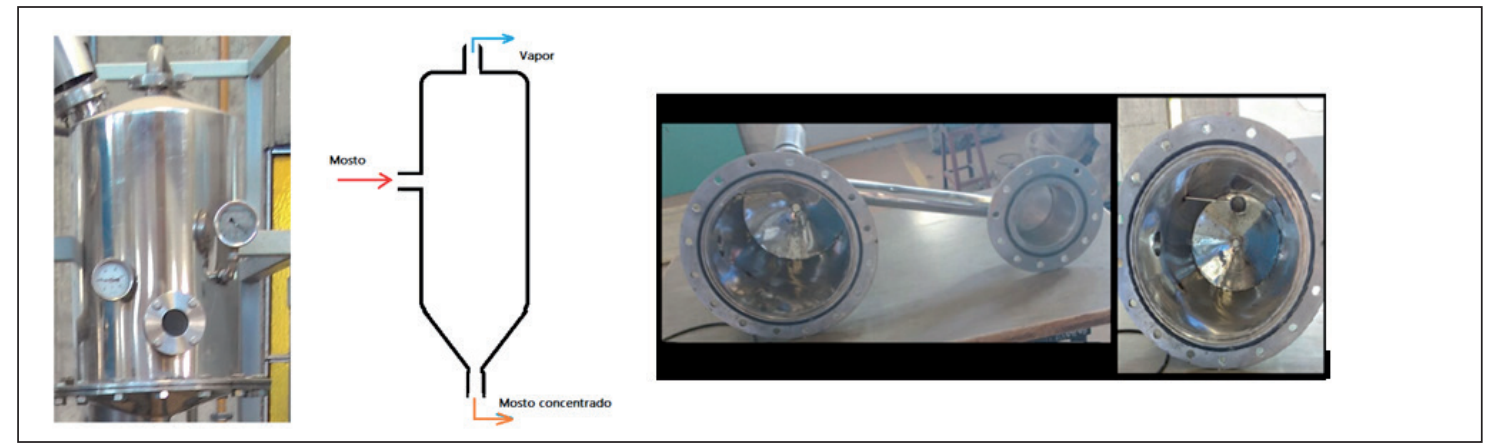

Figura $\mathbf{N}^{\circ}$ 1: Esquema del separador Flash.

Condensador y bomba de vacío: el vapor utilizado para calefaccionar el primer efecto, junto con el vapor del segundo efecto y el que proviene del separador flash al salir ingresa a un intercambiado de tubos en donde se condensa al ser enfriado con agua. A través de la bomba de vacío se extrae el condensado generando además una disminución de presión en el segundo separador flash.

Cabezal de alimentación al intercambiador de calor: El intercambiador es de tubos ascendentes por lo que el mosto ingresa al cabezal y sube por los tubos en donde se produce el intercambio.

Al trabajar con el equipo se observó que para poder limpiar el equipo es necesario colocarle una purga ya que el cabezal queda cargado con el producto.

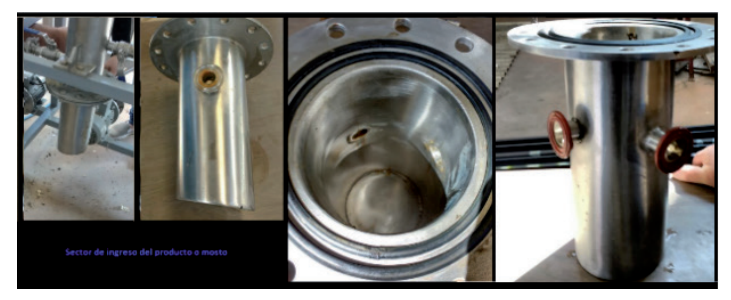

Figura 2: Cabezal de alimentación del intercambiador.

Considerando que el equipo no posee aislación, se trabajó realizando pruebas y se determinaron los puntos en los cuales se necesitaban los sensores.

\section{Conclusiones}

Analizando el funcionamiento del equipo, se determinaron las siguientes mejoras:

Variable a controlar: se determinó que la variable a controlar que más afecta la calidad final del producto es la temperatura.

Colocación de purga: Pudo observarse que la cámara de ingreso del mosto, del intercambiador de tubos verticales de película ascendente no posee purga para extraer el remanente de la operación.

Se determinó colocar las purgas correspondientes ya que de lo contrario se debería desarmar el cabezal para vaciado completo del producto y limpieza del mismo.

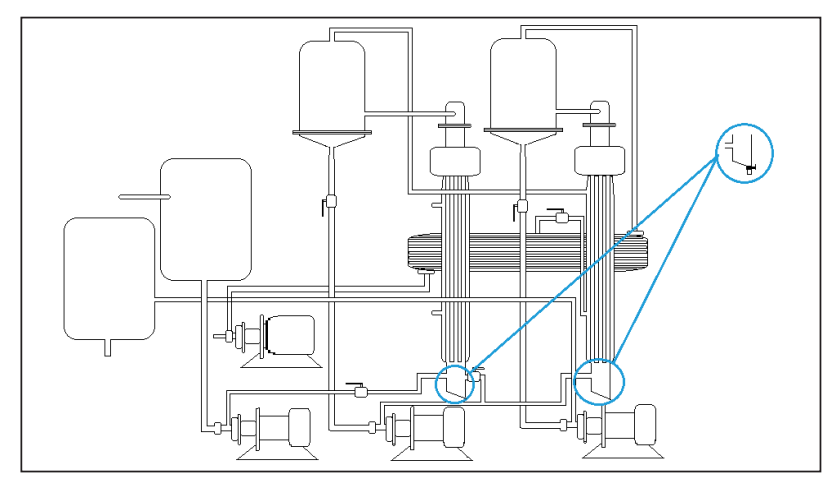

Figura 3: Ubicación de las purgas en el evaporador.

Colocación de válvula en la cañería de alimentación al segundo efecto: esto permite, de ser necesario, trabajar con un solo efecto o en paralelo, en el caso en el cual se alcance la concentración deseada o se deseen realizar tareas de investigación.

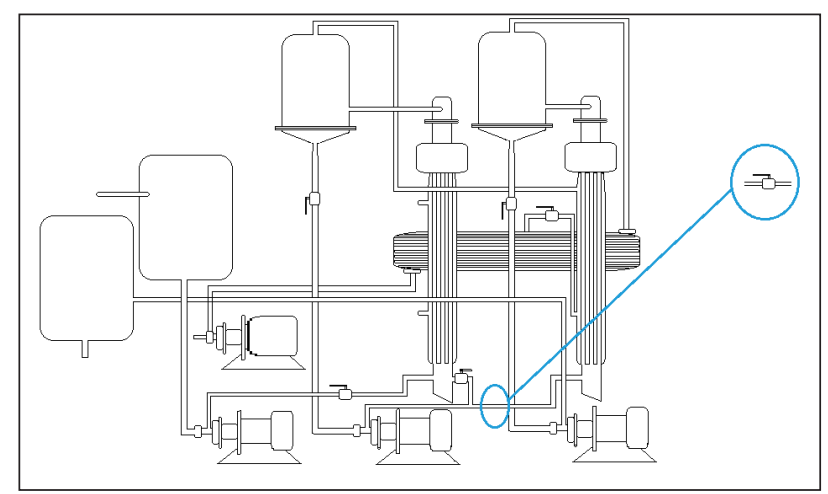

Figura 4: Ubicación de la valvula.

Disposición de los sensores: en función de las pérdidas de calor que se generan a través de las cañerías, se seleccionaron los puntos a censar. Los mismos pueden observarse en la figura $\mathrm{N}^{\circ}$ 5. Para la selección de los sensores se tomo como base la bibliografía: "Instrumentación y Control Avanzado de Procesos" Acedo (2006) e "Instrumentación Industrial" Creus Sole (2006). 


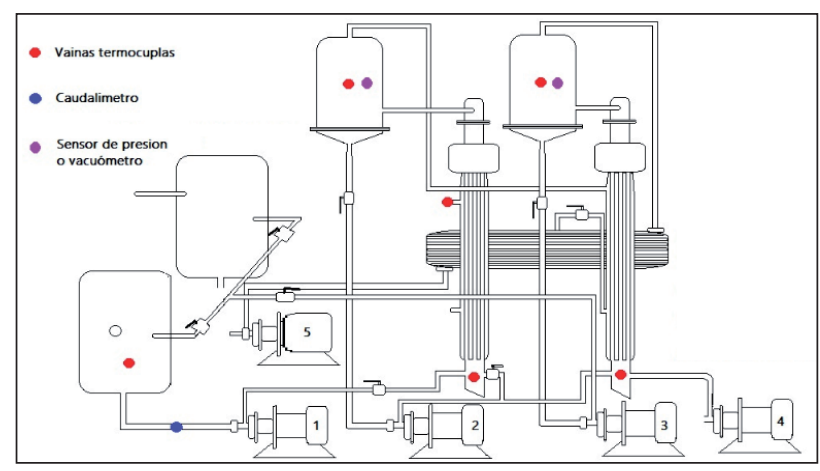

Figura 5: Ubicación de los sensores.

Referencias

1. Acedo Sánchez, José. Instrumentación y Control Avanzado de Procesos. Madrid, España. Editorial Díaz de Santos. 2006.

2. Cao, Eduardo. Transferencia de Calor en Ingeniería de Procesos. $2^{\mathrm{a}}$ edición. Argentina. Librería Editorial Nueva. 2004.
3. Creus Sole, Antonio. Instrumentación Industrial. $7^{\mathrm{a}}$ edición. México. Editorial Alfaomega. 2006. 10.

4. INV (2012). Estadísticas de exportaciones, recuperado 5/11/2012 de: http://www.inv.gov.ar/PDF/Estadisticas/ Exportaciones/2012/ProvinciaOrigenAgosto2012. pdf

5. Kern Donald. Procesos de Transferencia de Calor. México. Editorial CECSA. 1999.

6. Perry, Green, Maloney. Manual del Ingeniero Químico. $7^{\mathrm{a}}$ edición. México McGrawHill.1992.

7. TLV- Compañía especialista en vapor, recuperada 15/10/2012 de: http://www.tlv.com/global/LA/calculator/steam-table-temperature.html 